\title{
Spatial ordering and abnormal optical activity of DNA liquid-crystalline dispersion particles
}

\author{
S.V. Semenov ${ }^{2, *}$, and Yu.M. Yevdokimov ${ }^{1}$ \\ ${ }^{1}$ Engelhardt Institute of Molecular Biology RAS, 119991 Moscow, Russia \\ ${ }^{2}$ National Research Center "Kurchatov Institute", 123182 Moscow, Russia
}

\begin{abstract}
In our work, we investigate physicochemical and optical properties of double-strand DNA dispersions. The study of these properties is of biological interest, because it allows one to describe the characteristics of certain classes of chromosomes and DNA containing viruses. The package pattern of DNA molecules in the dispersions particles (DP) is examined. The consideration of the DNA liquid-crystalline DP optical activity based on the theory of electromagnetic wave absorption by large molecular aggregates has been performed. The investigation is also focused on various effects induced by the interaction between biological active compounds and DNA in the content of liquid-crystalline DP.
\end{abstract}

The nature of ds DNA dispersions properties is interesting from at least two points of view. From the theoretical point of view, the interest is caused by the fact that the physicochemical properties of dispersion particles with size $100-1,000 \AA$ can significantly differ from typical continuous solid phases' properties. The difference in the properties evolves as a result of "size effect". Such an effect is caused by the free energy attributed to the surface tension of particles and the existence of possible defects in molecule packing in the particles. The physicochemical properties of liquid-crystalline dispersions of nucleic acids are of biological interest because such particles open a gateway to describing the properties of Protozoan chromosomes and DNA-containing viruses, which are isolated microscopic systems with an ordered but labile packing. It is well known that the phase exclusion of the low molecular mass, rigid, linear, ds DNA (molecular mass $<1 \times 10^{6} \mathrm{Da}$ ) is realized under intensive mixing (stirring) of equal volumes the water-salt solutions containing poly(ethyleneglycol), PEG, with water-salt solutions containing a very low (5$10 \mu \mathrm{g} \mathrm{ml}^{-1}$ ) concentration of ds DNA at room temperature. As a result the formation of the liquid-crystalline dispersions (LCDs) of these molecules takes place [1]. The particles of the low molecular mass ds DNA dispersions are microscopic droplets of concentrated DNA solution, which cannot be taken in hand (The mode of the ds DNA packing in the LCD particles can model the packing of ds DNA in the primitive biological objects above). Considering the properties of the LCD particles we should keep in mind the following facts: 1) The polymer (PEG) is not included in the content of the formed particles. 2) The size (diameter) of the ds DNA LCD particle is determined by a fine balance between the free energy of these particles and their surface free energy. 3) The packing density of ds DNA molecules in the particles of a dispersion prepared by of mixing of DNA and PEG solutions is determined by the lateral interaction between neighboring DNA molecules at

* Corresponding author: Semenov_SV@nrcki.ru 
the moment of their close approaching. The distance between ds DNA molecules is determined by a balance between the repulsive intermolecular forces, and the compressing osmotic pressure of the PEG solution. The minimization of the excluded volume of neighboring linear, rigid, ds DNA molecules induces the parallel, unidirectional (nematiclike) alignment of ds DNA molecules. 4) The maximal density of ds DNA molecules in the LCD particles can be achieved under hexagonal packing. However, this is not a true crystal, because there are disordered water molecules between the ds DNA molecules. The ds DNA molecules possess some disorder around their positions; they can slide and bent with respect of each other, as well as they can rotate around their long axis. It corresponds to the liquid character of their packing. In hexagonal structure (as well as in a case of a hexagonal phase which is formed as a result of an increase in the DNA concentration in a water-salt solutions), we admit the existence of three kinds of orientationally ordered molecular layers. Double-stranded DNA molecules are oriented in a plane of these layers, whose thickness is on the order to the inter-molecular spacing. 5) The ds DNA molecules possess of several levels of chirality (helical secondary structure of ds DNA molecules, helical distribution of counter-ions near DNA surface as well as asymmetry of C-atoms in the sugar residues). Therefore, chiral and anisotropic properties of ds DNA molecules favor to helical twisting of the adjacent DNA molecules packed into the particles of the dispersion. Hence, unidirectional alignment of the ds DNA molecules compete with the tendency of these molecules to form spatially twisted structure of the LCDs. Here one can remind old statement made by Vainshtein and Chistyakov [2], that "we cannot describe directly the spatial distortions of molecules in three dimensions (only by X-ray scattering for liquid crystals) and indirect methods can be used to take into account rotational freedom of molecules about their long axes". To describe the ds DNA molecules spatial packing in LCD we used the small-angle X-ray scattering [3] and also the circular dichroism (CD) [4].

Theoretical calculations of CD of the ds DNA LCD particles is reasonable to perform by means of the approach based on the consideration of electromagnetic wave absorption by large molecular aggregates (MA) $[4,5]$. The optical properties of ds DNA LCD, considered as MA, are determined by long-distance interactions between chromophores in a given system. To study the optical characteristics of the structure, a ds DNA LCD particle can be divided into cells and each cell can be considered as a single chromophore. These efficient chromophores, composed by the DNA nitrogen base pairs absorbing dipoles, have an ordering similar to a cholesteric helix with the pitch P. The electric field at the point where a particular chromophore occurs is a superposition of the field of incident light and the electric fields generated by induced dipoles of all other chromophores of the system. As a result, light absorption is a process that involves the total molecular aggregate, and the electric field within the particle is obtained by solving a self-consistent system of the linear equations relative to the field amplitudes at each efficient chromophore. The full extinction cross section, necessary for the CD computations, is given by the optical theorem. To determine vector scattering amplitude the Maxwell's equations for the system under examination are directly solved, applying free-space tensor Green's function. As a result the obtained expression for extinction cross section gives the possibility to calculate the $C D$ of ds DNA LCD as a function of different physical parameters, such as pitch $\mathrm{P}$, particle size $\mathrm{D}$, etc., as well as the change in ordering due to DNA interaction with golden nanoparticles $[4,5]$. It allows one to describe the peculiarities of the CD of ds DNA LCD complexes with different antibiotics, which is essential for creation of biosensors.

\section{References}

1. Yu.M. Yevdokimov, V.I. Salyanov, S.V. Semenov, S.G. Skuridin, DNA LiquidCrystalline Dispersions and Nanoconstructions (CRC Press, New York, 2011)

2. L.V. Azároff, Mol. Cryst. Liq. Cryst. 60, 73 (1980)

3. E.V. Shtykova et al., Eur. Biohys. J. 39, 1313 (2010)

4. S.V. Semenov, Yu.M. Yevdokimov, Biophysics 60, 188 (2015)

5. D. Keller, C. Bustamante, J. Chem. Phys. 84, 2961 (1986) 